Homology, Homotopy and Applications, vol. 7(3), 2005, pp.83-98

\title{
COMPUTING THE EQUIVARIANT EULER CHARACTERISTIC OF ZARISKI AND ÉTALE SHEAVES ON CURVES
}

\author{
BERNHARD KÖCK \\ (communicated by J.F. Jardine)
}

\begin{abstract}
We prove an equivariant Grothendieck-Ogg-Shafarevich formula. This formula may be viewed as an étale analogue of well-known formulas for Zariski sheaves generalizing the classical Chevalley-Weil formula. We give a new approach to those formulas (first proved by Ellingsrud/Lønsted, Nakajima, Kani and Ksir) which can also be applied in the étale case.
\end{abstract}

Dedicated to Victor Snaith on the occasion of his 60-th birthday.

\section{Introduction}

This paper deals with the Riemann-Roch problem for equivariant Zariski sheaves and equivariant étale sheaves on smooth projective curves, i.e. with the computation of their (equivariant!) Euler characteristic. In the case of Zariski sheaves we give a new, very natural and quick approach to generalizations of the classical Chevalley-Weil formula proved by Ellingsrud/Lønsted (see [EL]), Kani (see [Ka]) and Nakajima (see [Na]) in the 1980s and we derive generalizations of a more recent result of Ksir (see $[\mathbf{K s}]$ ). In the case of étale sheaves we develop and prove an equivariant Grothendieck-Ogg-Shafarevich formula by imitating our new approach for Zariski sheaves.

Let $X$ be a connected smooth projective curve over an algebraically closed field $k$ and let $G$ be a finite subgroup of $\operatorname{Aut}(X / k)$ of order $n$. We assume throughout this paper that the canonical projection $\pi: X \rightarrow Y:=X / G$ is tamely ramified.

Using the coherent Lefschetz fixed point formula (see [Do1] or [BFQ] or [Kö1]) in conjunction with the Riemann-Roch formula and Hurwitz formula, we prove the following theorem (see Theorem 1.1) which effectively implies all known formulas (see Corollaries 1.3, 1.4, 1.7 and 1.8) for the equivariant Euler characteristic

$$
\chi(G, X, \mathcal{E}):=\left[H^{0}(X, \mathcal{E})\right]-\left[H^{1}(X, \mathcal{E})\right]
$$

of a locally free $G$-sheaf $\mathcal{E}$ on $X$, considered as an element of the Grothendieck group $K_{0}(G, k)$ of all $k$-representations of $G$.

Received November 3, 2004, revised April 6, 2005; published on November 12, 2005. 2000 Mathematics Subject Classification: 14F20, 14L30, 14 H30.

Key words and phrases: Equivariant Euler characteristic, étale cohomology, Grothendieck-Ogg-

Shafarevich formula, conductor, Lefschetz formula, Riemann-Roch formula, Hurwitz formula.

(C) 2005, Bernhard Köck. Permission to copy for private use granted. 


\section{Theorem 1.}

$$
\chi(G, X, \mathcal{E})=\left(\left(1-g_{Y}\right) r+\frac{1}{n} \operatorname{deg}(\mathcal{E})\right)[k[G]]-\frac{1}{n} \sum_{P \in X} \sum_{d=0}^{e_{P}-1} d\left[\operatorname{Ind}_{G_{P}}^{G}\left(\mathcal{E}(P) \otimes \chi_{P}^{d}\right)\right] .
$$

Here, $r$ denotes the rank of $\mathcal{E}, \mathcal{E}(P)$ the fibre of $\mathcal{E}$ at $P$ and $\chi_{P}$ the character of $G_{P}$ which is given by the representation of $G_{P}$ on the cotangent space $\mathfrak{m}_{P} / \mathfrak{m}_{P}^{2}$.

We now pass to the étale world. We fix a prime $l \neq \operatorname{char}(k)$. Let $\mathcal{F}$ be a constructible $\mathbb{F}_{l}$-sheaf on the étale site $X_{\text {ét }}$ which carries a $G$-action compatible with the given $G$-action on $X$. We are interested in computing the equivariant Euler characteristic

$$
\chi_{\text {ét }}(G, X, \mathcal{F}):=\left[H_{\text {ét }}^{0}(X, \mathcal{F})\right]-\left[H_{\text {ét }}^{1}(X, \mathcal{F})\right]+\left[H_{\text {ét }}^{2}(X, \mathcal{F})\right]
$$

considered as an element of the Grothendieck group $K_{0}\left(G, \mathbb{F}_{l}\right)$ of $\mathbb{F}_{l}$-representations of $G$.

In the extreme case that $G$ is the trivial group, this problem is solved by the classical Grothendieck-Ogg-Shafarevich formula (see Theorem 2.12 on p. 190 in [Mi]). In the extreme case that $\mathcal{F}$ is the constant sheaf with trivial $G$-action, a satisfactory answer to this problem follows from Remark 2.9 on p. 187 in [Mi] (see Remark 2.2(b)). By imitating our approach for Zariski sheaves we prove the following result for an arbitrary group $G$ and arbitrary sheaf $\mathcal{F}$ (see Theorem 2.1):

Theorem 2. We assume that the characteristic of $k$ does not divide $n$. Then we have:

$$
\begin{aligned}
& \chi_{\text {ét }}(G, X, \mathcal{F})= \\
& =\left(\left(2-2 g_{Y}\right) \operatorname{dim}_{\mathbb{F}_{l}}\left(\mathcal{F}_{\bar{\eta}}\right)-\frac{1}{n} \alpha(\mathcal{F})+\sum_{P \in X} \frac{e_{p}}{n}\left(\operatorname{dim}_{\mathbb{F}_{l}}\left(\mathcal{F}_{P}\right)-\operatorname{dim}_{\mathbb{F}_{l}}\left(\mathcal{F}_{\bar{\eta}}\right)\right)\right)\left[\mathbb{F}_{l}[G]\right] \\
& \quad-\sum_{P \in X} \frac{e_{P}}{n}\left[\operatorname{Ind}_{G_{P}}^{G}\left(\mathcal{F}_{P} \otimes I_{P}\right)\right] .
\end{aligned}
$$

Here, $g_{Y}$ denotes the genus of $Y:=X / G, \eta$ the generic point of $X, \alpha(\mathcal{F}):=$ $\sum_{P \in X} \alpha_{P}(\mathcal{F})$ the sum of the wild conductors of $\mathcal{F}, e_{P}$ the ramification index of the canonical projection $\pi: X \rightarrow Y$ at $P \in X$ and $I_{P}:=\operatorname{ker}\left(\mathbb{F}_{l}\left[G_{P}\right] \rightarrow \mathbb{F}_{l}\right)$ the augmentation representation of the decomposition group $G_{P}$ at $P$.

As a corollary, we obtain that $\alpha(\mathcal{F})$ is divisible by $n$ (see Corollary 2.4). Furthermore, this formula has the following simple shape, if $\pi$ is étale (see Remark $2.2(\mathrm{a}))$ :

$$
\chi_{\text {ét }}(G, X, \mathcal{F})=\frac{1}{n} \chi_{\text {ét }}(X, \mathcal{F})\left[\mathbb{F}_{l}[G]\right] .
$$

In fact, this formula is valid without the assumption that $\operatorname{char}(k)$ does not divide $n$. In particular, the (non-equivariant) Euler characteristic $\chi_{\text {ét }}(X, \mathcal{F})$ is divisible by $n$.

Acknowledgments. I would like to thank Igor Zhukov for raising the equivariant Riemann-Roch problem for étale sheaves and for drawing my attention to the paper [Do2]. Furthermore, I would like to thank him and Victor Snaith for helpful and 
encouraging discussions, for reading carefully a preliminary version of this paper and for suggesting several corrections.

\section{The formulas of Ellingsrud/Lønsted, Nakajima, Kani and Ksir revisited}

The object of this section is to give a new approach to generalizations of the classical Chevalley-Weil formula published by Ellingsrud/Lønsted, Nakajima and Kani and to derive generalizations of a comparatively simple formula recently published by Ksir.

Let $X$ be a connected smooth projective curve over an algebraically closed field $k$ and let $G$ be a finite subgroup of $\operatorname{Aut}(X / k)$ of order $n$. We assume in this section that the canonical projection $\pi: X \rightarrow Y:=X / G$ is tamely ramified.

We denote the genus of $X$ and $Y$ by $g_{X}$ and $g_{Y}$, respectively. For any (closed) point $P \in X$, let $G_{P}:=\{\sigma \in G: \sigma(P)=P\}$ denote the decomposition group and let $e_{P}$ denote the ramification index of $\pi$ at $P$. It is well-known (see Corollaire 1 of Proposition 7, Chapitre IV on p. 75 of [Se1]) that $G_{P}$ is a cyclic group of order $e_{P}$ and that

$$
\operatorname{Hom}\left(G_{P}, k^{\times}\right)=\left\{\chi_{P}^{0}, \ldots, \chi_{P}^{e_{P}-1}\right\}
$$

where $\chi_{P}: G_{P} \rightarrow k^{\times}$denotes the character which is given by the action of $G_{P}$ on the cotangent space $\mathfrak{m}_{P} / \mathfrak{m}_{P}^{2}$. For $Q \in Y$ we set $e_{Q}:=e_{P}$ where $P \in \pi^{-1}(Q)$.

We denote the Grothendieck group of all $k$-representations of $G$ (of finite dimension) by $K_{0}(G, k)$. It is free with basis $\hat{G}$ where $\hat{G}$ denotes the set of isomorphism classes of irreducible $k$-representations of $G$.

Now, let $\mathcal{E}$ be a locally free $G$-module on $X$ of rank $r$, i.e., we have $\mathcal{O}_{X^{-}}$ isomorphisms $g^{*}(\mathcal{E}) \rightarrow \mathcal{E}, g \in G$, which satisfy the usual composition rules. Then, the Zariski cohomology groups $H^{i}(X, \mathcal{E}), i \geqslant 0$, are $k$-representations of $G$. Let

$$
\chi(G, X, \mathcal{E}):=\left[H^{0}(X, \mathcal{E})\right]-\left[H^{1}(X, \mathcal{E})\right] \in K_{0}(G, k)
$$

denote the equivariant Euler characteristic of $X$ with values in $\mathcal{E}$. For any $P \in X$, we view the fibre $\mathcal{E}(P):=\mathcal{E}_{P} / \mathfrak{m}_{P} \mathcal{E}_{P}$ as a $k$-representation of $G_{P}$.

The following theorem computes the equivariant Euler characteristic $\chi(G, X, \mathcal{E})$.

Theorem 1.1. We have in $K_{0}(G, k)$ :

$$
n \cdot \chi(G, X, \mathcal{E})=\left(n\left(1-g_{Y}\right) r+\operatorname{deg}(\mathcal{E})\right)[k[G]]-\sum_{P \in X} \sum_{d=0}^{e_{P}-1} d\left[\operatorname{Ind}_{G_{P}}^{G}\left(\mathcal{E}(P) \otimes \chi_{P}^{d}\right)\right] .
$$

Proof. By classical representation theory (see Corollary (17.10) on p. 424 in [CR2]) it suffices to show that the Brauer characters of both sides of formula (1) coincide. For any $k$-representation $V$ of $G$ and for any $\sigma \in G$ of order prime to $\operatorname{char}(k)$ we write $\operatorname{Trace}(\sigma \mid V)$ for the value of the Brauer character of $V$ at $\sigma$. Recall that

$$
\operatorname{Trace}(\sigma \mid V)=\sum_{i=1}^{\operatorname{dim}(V)} \varphi\left(\alpha_{i}\right)
$$


where $\alpha_{i}, i=1, \ldots, \operatorname{dim}(V)$, are the eigenvalues of the $k$-linear map $\sigma$ on $V$ and $\varphi: k^{\times} \rightarrow K^{\times}$is the Teichmüller character from the group of invertible elements in $k$ to the group of invertible elements in the quotient field $K:=Q(W(k))$ of the Witt ring $W(k)$ of $k$. (We set $K:=k$ and $\varphi:=$ id, if $p=0$.)

Let $\sigma \in G$ such that $\operatorname{char}(k)$ does not divide the order of $\sigma$ and let $X^{\sigma}:=\{P \in X$ : $\sigma(P)=P\}$ denote the set of points in $X$ fixed by $\sigma$. Then we have:

$$
\begin{aligned}
& \sum_{P \in X} \sum_{d=0}^{e_{P}-1} d \cdot \operatorname{Trace}\left(\sigma \mid \operatorname{Ind}_{G_{P}}^{G}\left(\mathcal{E}(P) \otimes \chi_{P}^{d}\right)\right)= \\
& =\sum_{P \in X} \sum_{d=0}^{e_{P}-1} \frac{d}{e_{P}} \sum_{\tau \in G, \tau^{-1} \sigma \tau \in G_{P}} \operatorname{Trace}\left(\tau^{-1} \sigma \tau \mid \mathcal{E}(P)\right) \cdot \chi_{P}^{d}\left(\tau^{-1} \sigma \tau\right)
\end{aligned}
$$

(by Lemma (21.28) on p. 509 in [CR2])

$=\sum_{P \in X} \sum_{d=0}^{e_{P}-1} \frac{d}{e_{P}} \sum_{\tau \in G, \tau(P) \in X^{\sigma}} \operatorname{Trace}(\sigma \mid \mathcal{E}(\tau(P))) \cdot \chi_{\tau(P)}^{d}(\sigma)$

$=\sum_{P \in X^{\sigma}} \frac{n}{e_{P}} \cdot \operatorname{Trace}(\sigma \mid \mathcal{E}(P)) \cdot \sum_{d=0}^{e_{P}-1} d \cdot \chi_{P}^{d}(\sigma)$

$= \begin{cases}n \cdot r \cdot \sum_{P \in X} \frac{e_{P}-1}{2} & \text { for } \sigma=\mathrm{id} \\ n \cdot \sum_{P \in X^{\sigma}} \operatorname{Trace}(\sigma \mid \mathcal{E}(P)) \cdot\left(\chi_{P}(\sigma)-1\right)^{-1} & \text { for } \sigma \neq \mathrm{id} \quad \text { (see Lemma 1.2 below) }\end{cases}$

For $\sigma \neq$ id we have Trace $(\sigma \mid k[G])=0$, so the character value of the right hand side of formula (1) at the place $\sigma$ equals

$$
n \cdot \sum_{P \in X^{\sigma}} \operatorname{Trace}(\sigma \mid \mathcal{E}(P)) \cdot\left(1-\chi_{P}(\sigma)\right)^{-1} .
$$

By the Lefschetz fixed point formula (see Example 3 in [Kö1] or [BFQ] or [Do1]; here we use the assumption that $\operatorname{char}(k)$ does not divide ord $(\sigma))$, this equals the character value of the left hand side at the place $\sigma$.

For $\sigma=$ id we have:

$$
\begin{aligned}
& \left(n\left(1-g_{Y}\right) r+\operatorname{deg}(\mathcal{E})\right) \cdot \operatorname{Trace}(\sigma \mid k[G])= \\
& =n\left(n\left(1-g_{Y}\right) r+\operatorname{deg}(\mathcal{E})\right) \\
& =n\left(\left(1-g_{X}\right) r+\operatorname{deg}(\mathcal{E})+r \sum_{P \in X} \frac{e_{P}-1}{2}\right)
\end{aligned}
$$

by the Hurwitz formula (see Corollary 2.4 on p. 301 in [Ha]). Hence, the character value of the right hand side of formula (1) at the place $\sigma=$ id equals

$$
n\left(\left(1-g_{X}\right) r+\operatorname{deg}(\mathcal{E})\right) \text {. }
$$

By the Riemann-Roch formula (see $\S 1$ in Chapter IV of [Ha] and Exercise 6.11 on p. 149 in $[\mathbf{H a}]$ ), this equals the character value of the left hand side at the place $\sigma=$ id.

Thus, the proof of Theorem 1.1 is complete. 
Lemma 1.2. Let $m \in \mathbb{N}$ and $\zeta \neq 1$ an $m$-th root of unity. Then we have:

$$
m(\zeta-1)^{-1}=\sum_{d=1}^{m-1} d \zeta^{d}
$$

Proof. $\left(\sum_{d=1}^{m-1} d \zeta^{d}\right)(\zeta-1)=\sum_{d=1}^{m-1} d \zeta^{d+1}-\sum_{d=1}^{m-1} d \zeta^{d}=(m-1) \zeta^{m}-\sum_{d=1}^{m-1} \zeta^{d}=$ $m$.

Remark. A generalization of Theorem 1.1 and the subsequent Corollary 1.4 to the so-called weakly ramified case can be found in [Kö2].

The following corollary is the main result of the paper [EL] by Ellingsrud and Lønsted; it computes the multiplicity of any irreducible representation $V \in \hat{G}$ in the equivariant Euler characteristic $\chi(G, X, \mathcal{E})$, if $\operatorname{char}(k)$ does not divide $n$. While the proof of Ellingsrud and Lønsted is based on the study of the cokernel of the natural embedding $\pi^{*}\left(\pi_{*}^{G}(\mathcal{E})\right) \hookrightarrow \mathcal{E}$, we derive it from Theorem 1.1 and hence from the Lefschetz fixed point formula.

Corollary 1.3 (Formula (3.7) in [EL]). We assume that char $(k)$ does not divide n. For $Q \in Y, d \in \mathbb{N}$ and $V \in \hat{G}$, let $n_{d, Q, \mathcal{E}, V}$ denote the multiplicity of $\chi_{P}^{d}$ in $\mathcal{E}(P) \otimes \operatorname{Res}_{G_{P}}^{G}(V)$ where $P \in \pi^{-1}(Q)$. Then we have in $K_{0}(G, k)_{\mathbb{Q}}$ :

$$
\chi(G, X, \mathcal{E})=\left(\frac{1}{n} \operatorname{deg}(\mathcal{E})+\left(1-g_{Y}\right) r\right)[k[G]]-\sum_{V \in \hat{G}} \sum_{Q \in Y} \sum_{d=1}^{e_{Q}}\left(1-\frac{d}{e_{Q}}\right) n_{d, Q, \mathcal{E}, \check{V}}[V] .
$$

Proof. Let $\langle\rangle:, K_{0}(G, k) \times K_{0}(G, k) \rightarrow \mathbb{Z}$ denote the usual character pairing. Then, for all $P \in X$ and $d \in\left\{0, \ldots, e_{P}-1\right\}$, we have:

$$
\begin{aligned}
& \sum_{V \in \hat{G}}\left\langle\left[\chi_{P}^{d}\right],\left[\mathcal{E}(P) \otimes \operatorname{Res}_{G_{P}}^{G}(\check{V})\right]\right\rangle[V]= \\
= & \sum_{V \in \hat{G}}\left\langle\left\langle\left[\operatorname{Ind}_{G_{P}}^{G}\left(\mathcal{E}(P) \otimes \chi_{P}^{-d}\right)\right],[V]\right\rangle[V]\right. \\
= & {\left[\operatorname{Ind}_{G_{P}}^{G}\left(\mathcal{E}(P) \otimes \chi_{P}^{-d}\right)\right] \quad \text { in } \quad K_{0}(G, k) . }
\end{aligned}
$$

Hence we have:

$$
\begin{aligned}
& \sum_{V \in \hat{G}} \sum_{Q \in Y} \sum_{d=1}^{e_{Q}}\left(1-\frac{d}{e_{Q}}\right) n_{d, Q, \mathcal{E}, \check{V}}[V]= \\
& =\sum_{P \in X} \frac{e_{P}}{n} \sum_{d=1}^{e_{P}-1}\left(1-\frac{d}{e_{P}}\right) \sum_{V \in \hat{G}}\left\langle\left[\chi_{P}^{d}\right],\left[\mathcal{E}(P) \otimes \operatorname{Res}_{G_{P}}^{G}(\check{V})\right]\right\rangle[V] \\
& =\frac{1}{n} \sum_{P \in X} \sum_{d=1}^{e_{P}-1}\left(e_{P}-d\right)\left[\operatorname{Ind}_{G_{P}}^{G}\left(\mathcal{E}(P) \otimes \chi_{P}^{-d}\right)\right] \\
& =\frac{1}{n} \sum_{P \in X} \sum_{d=1}^{e_{P}-1} d\left[\operatorname{Ind}_{G_{P}}^{G}\left(\mathcal{E}(P) \otimes \chi_{P}^{d}\right)\right] .
\end{aligned}
$$

Thus, Corollary 1.3 follows from Theorem 1.1. 
The following corollary is the main result of the paper [Na] by Nakajima. Part (a) of it has also been proved by Kani (see Theorem 2 in $[\mathbf{K a}]$ ). In addition to Theorem 1.1 we use the facts that the Euler characteristic $\chi(G, X, \mathcal{E})$ is an element of the Grothendieck group $K_{0}(k[G])$ of projective $k[G]$-modules (see Theorem 1 in $[\mathrm{Na}]$ or Remark 1.5(a) below) and that the Cartan homomorphism $K_{0}(k[G]) \rightarrow$ $K_{0}(G, k)$ is injective. The corollary expresses the Euler characteristic $\chi(G, X, \mathcal{E})$ as an integral linear combination of certain projective $k[G]$-modules. Our proof shortens the somewhat lengthy calculations in $[\mathbf{N a}]$.

Corollary 1.4 (Theorem 2 in [Na])).

(a) There is a projective $k[G]$-module $N_{G, X}$ (which is unique up to isomorphism) such that

$$
\stackrel{n}{\oplus} N_{G, X} \cong \underset{P \in X}{\oplus} \underset{d=0}{\stackrel{e_{P}-1}{\oplus}} \stackrel{d}{\oplus} \operatorname{Ind}_{G_{P}}^{G}\left(\chi_{P}^{d}\right) .
$$

(b) For any $P \in X$, let $l_{P, 1}, \ldots, l_{P, r} \in\left\{0, \ldots, e_{P}-1\right\}$ be given by the equation

$$
[\mathcal{E}(P)]=\sum_{i=1}^{r}\left[\chi_{P}^{-l_{P, i}}\right] \quad \text { in } \quad K_{0}\left(G_{P}, k\right)
$$

furthermore, for any $Q \in Y$, we fix a point $\tilde{Q} \in X$ with $\pi(\tilde{Q})=Q$. Then we have:

$$
\chi(G, X, \mathcal{E}) \equiv-r\left[N_{G, X}\right]+\sum_{Q \in Y} \sum_{i=1}^{r} \sum_{d=1}^{l_{\tilde{Q}, i}}\left[\operatorname{Ind}_{G_{\tilde{Q}}}^{G}\left(\chi_{\tilde{Q}}^{-d}\right)\right] \text { in } K_{0}(k[G]) \bmod \mathbb{Z}[k[G]] .
$$

Proof.

(a) Applying Theorem 1.1 to the sheaf $\mathcal{E}=\mathcal{O}_{X}$ with trivial $G$-action, we obtain the following equality in $K_{0}(G, k)$ and hence in $K_{0}(k[G])$ :

$$
n \cdot \chi\left(G, X, \mathcal{O}_{X}\right)=n\left(1-g_{Y}\right)[k[G]]-\sum_{P \in X} \sum_{d=0}^{e_{P}-1} d\left[\operatorname{Ind}_{G_{P}}^{G}\left(\chi_{P}^{d}\right)\right] .
$$

This equality shows that the class of the projective $k[G]$-module $\oplus_{d=0}^{e_{P}-1} \oplus^{d} \operatorname{Ind}_{G_{P}}^{G}\left(\chi_{P}^{d}\right)$ is divisible by $n$ in $K_{0}(k[G])$. Writing the quotient as a linear combination of classes of indecomposable projective modules we see that the quotient is in fact the class of a projective $k[G]$-module, say $N_{G, X}$. This immediately implies part (a).

(b) We first prove the following congruence:

$$
\operatorname{deg}(\mathcal{E}) \equiv \sum_{P \in X} \sum_{i=1}^{r} l_{P, i} \bmod n
$$

For this, we may obviously assume that $r=1$. We write $l_{P}$ for $l_{P, 1}$. Let $\mathcal{K}$ denote the sheaf of meromorphic functions on $X$, i.e., the constant sheaf associated with the function field $K(X)$ of $X$. Then $\mathcal{E}$ is a $G$-subsheaf of the constant sheaf $\mathcal{E} \otimes \mathcal{K}$. But $\mathcal{E} \otimes$ $\mathcal{K}$ is isomorphic to $\mathcal{K}$ as a $G$-sheaf since the twisted group ring $K(X) G$ is isomorphic to the ring $M_{n}(K(Y))$ of $n \times n$-matrices over the function field $K(Y)$ by Galois theory and since there is (up to isomorphism) only one module of $K(Y)$-dimension $n$ over $M_{n}(K(Y))$. So, we may assume that $\mathcal{E}=\mathcal{O}_{X}(D)$ for some equivariant Weil 
divisor $D=\sum_{P \in X} n_{P}[P]$. Now it is easy to see that $l_{P} \equiv n_{p} \bmod e_{P}$ for all $P$. So, for any $Q \in Y$ we have: $\sum_{P \in \pi^{-1}(Q)} l_{P}=\frac{n}{e_{P}} l_{P} \equiv \frac{n}{e_{P}} n_{P}=\sum_{P \in \pi^{-1}(Q)} n_{P} \bmod n$. Thus, the congruence above is proved.

Hence, by Theorem 1.1, we have in $K_{0}(G, k) / n \mathbb{Z}[k[G]]$ :

$$
\begin{aligned}
& n \cdot \chi(G, X, \mathcal{E})= \\
& =\sum_{P \in X} \sum_{i=1}^{r} l_{P, i}[k[G]]-\sum_{P \in X} \sum_{i=1}^{r} \sum_{d=1}^{e_{P}-1} d\left[\operatorname{Ind}_{G_{P}}^{G}\left(\chi_{P}^{d-l_{P, i}}\right)\right] \\
& =\sum_{P \in X} \sum_{i=1}^{r} \operatorname{Ind}_{G_{P}}^{G}\left(\sum_{d=0}^{e_{P}-1} l_{P, i}\left[\chi_{P}^{d}\right]-\sum_{d=0}^{e_{P}-1} d\left[\chi_{P}^{d-l_{P, i}}\right]\right) \\
& =\sum_{P \in X} \sum_{i=1}^{r} \operatorname{Ind}_{G_{P}}^{G}\left(\sum_{d=0}^{e_{P}-1} \alpha_{P, i, d}\left[\chi_{P}^{d}\right]\right)
\end{aligned}
$$

where $\alpha_{P, i, d}:= \begin{cases}-d & \text { for } d=0, \ldots, e_{P}-l_{P, i}-1 \\ -d+e_{P} & \text { for } d=e_{P}-l_{P, i}, \ldots, e_{P}-1 .\end{cases}$

On the other hand, we have:

$$
\begin{aligned}
& -n \cdot r \cdot\left[N_{G, X}\right]+n \sum_{Q \in Y} \sum_{i=1}^{r} \sum_{d=1}^{l_{\tilde{Q}, i}}\left[\operatorname{Ind}_{G_{\tilde{Q}}}^{G}\left(\chi_{\tilde{Q}}^{-d}\right)\right]= \\
& =-r \sum_{P \in X} \sum_{d=0}^{e_{P}-1} d\left[\operatorname{Ind}_{G_{P}}^{G}\left(\chi_{P}^{d}\right)\right]+\sum_{P \in X} e_{P} \sum_{i=1}^{r} \sum_{d=1}^{l_{P, i}}\left[\operatorname{Ind}_{G_{P}}^{G}\left(\chi_{P}^{-d}\right)\right] \\
& =\sum_{P \in X} \sum_{i=1}^{r} \operatorname{Ind}_{G_{P}}^{G}\left(-\sum_{d=0}^{e_{P}-1} d\left[\chi_{P}^{d}\right]+\sum_{d=1}^{l_{P, i}} e_{P}\left[\chi_{P}^{e_{P}-d}\right]\right) \\
& =\sum_{P \in X} \sum_{i=1}^{r} \operatorname{Ind}_{G_{P}}^{G}\left(\sum_{d=0}^{e_{P}^{-1}} \alpha_{P, i, d}\left[\chi_{P}^{d}\right]\right) .
\end{aligned}
$$

Thus, Corollary 1.4 is proved.

\section{Remark 1.5.}

(a) In order to prove that $\chi(G, X, \mathcal{E})$ is in $K_{0}(k[G])$ for all locally free $G$-modules $\mathcal{E}$, it suffices to show that the element $\frac{1}{n} \sum_{P \in X} \sum_{d=0}^{e_{p}-1} d\left[\operatorname{Ind}_{G_{P}}^{G}\left(\chi_{P}^{d}\right)\right]$ is in $K_{0}(k[G])$ which in turn follows from the fact that $\chi(G, X, \mathcal{L})$ is in $K_{0}(k[G])$ for one invertible $G$ module $\mathcal{L}$ on $X$. (Apply twice the formula in part (b) of Corollary 1.4). If $\operatorname{deg}(\mathcal{L})>$ $2 g_{X}-2$, a nice and short proof of this fact using equivariant cohomology can be found in Borne's thesis (see Corollaire 3.14 on p. 61 in [Bo]).

(b) The equation

$$
\chi\left(G, X, \mathcal{O}_{X}\right)=\left(1-g_{Y}\right)[k[G]]-\left[N_{G, X}\right]
$$

occurring in the proof of part (a) may be considered as an equivariant version of the classical Hurwitz formula, see Théorème 3.16 on p. 62 in Borne's thesis [Bo]. He gives a proof of this formula and of part (a) of Corollary 1.4 which does not 
use the work of Nakajima or Kani either and whose main ingredient is the coherent Lefschetz fixed point formula as well.

The following example illustrates part (a) of Corollary 1.4; it has been proved in [Bo] directly using Hilbert 90 (see Proposition 3.7 on p. 56 in [Bo]).

Example 1.6. We assume that $e_{P}=n$ for all $P \in X_{\mathrm{ram}}:=\left\{P \in X: e_{P} \neq 1\right\}$. Let $\chi: G \rightarrow k^{\times}$be a character. We write $\chi=\chi_{P}^{a_{P}}$ for some $a_{P} \in \mathbb{N}$ (for all $P \in X_{\text {ram }}$ ). Then we have:

$$
\sum_{P \in X_{\mathrm{ram}}} a_{P} \equiv 0 \bmod n .
$$

The following corollary is a main result of the paper [Ka] by Kani; it generalizes the classical Chevalley-Weil formula.

Corollary 1.7 (Corollary of Theorem 2 in [Ka]). Let $\Omega$ denote the sheaf of holomorphic differentials on $X$. Then we have in $K_{0}(G, k)$ :

$$
\left[H^{0}(X, \Omega)\right]=[k]+\left(g_{Y}-1\right)[k[G]]+\left[\check{N}_{G, X}\right] .
$$

Proof. It is well-known that $\operatorname{deg}(\Omega)=2 g_{X}-2$ and that $\Omega(P)$ is $k\left[G_{P}\right]$-isomorphic to $\mathfrak{m}_{P} / \mathfrak{m}_{P}^{2}$ for all $P \in X$. Hence, by Theorem 1.1, we have in $K_{0}(G, k)$ :

$$
\begin{aligned}
& n \cdot \chi(G, X, \Omega)= \\
& =\left(n\left(1-g_{Y}\right)+\left(2 g_{X}-2\right)\right)[k[G]]-\sum_{P \in X} \sum_{d=0}^{e_{P}-1} d\left[\operatorname{Ind}_{G_{P}}^{G}\left(\chi_{P}^{d+1}\right)\right] \\
& =\left(n\left(1-g_{Y}\right)+n\left(2 g_{Y}-2\right)\right)[k[G]]-\sum_{P \in X} \sum_{d=0}^{e_{P}-1}\left(d+1-e_{P}\right)\left[\operatorname{Ind}_{G_{P}}^{G}\left(\chi_{P}^{d+1}\right)\right] \\
& =n\left(g_{Y}-1\right)[k[G]]+n\left[\check{N}_{G, X}\right] .
\end{aligned}
$$

Since $H^{1}(X, \Omega)$ is isomorphic to the trivial representation $k$, this proves Corollary 1.7 .

The following corollary generalizes a recently published result of Ksir (see $[\mathbf{K s}]$ ). While we derive it from the previous corollary, her proof is much more elementary. To be more precise: While our proof is based on the Lefschetz fixed point formula (see the proof of Theorem 1.1), her proof uses only the Riemann-Roch and Hurwitz theorem and some elementary character theory. However, her proof seems to work only in the case that not only the representation $V$, but all (irreducible) $k$ representations of $G$ are rationally valued. Here, we call a $k[G]$-module rationally valued if its (Brauer) character takes only rational values. For each point $Q \in Y$, we fix a point $\tilde{Q} \in X$ in the fibre $\pi^{-1}(Q)$.

Corollary 1.8. We assume that $\operatorname{char}(k)$ does not divide $n$. Let $V$ be a non-trivial rationally valued irreducible $k$-representation of $G$. Then the multiplicity of $V$ in the $k$-representation $H^{0}\left(X, \Omega_{X}\right)$ is equal to

$$
\operatorname{dim}(V)\left(g_{Y}-1\right)+\frac{1}{2} \sum_{Q \in Y}\left(\operatorname{dim}(V)-\operatorname{dim}\left(V^{G_{\tilde{Q}}}\right)\right) .
$$


Proof. This follows from Corollary 1.7, the following Proposition 1.9 and the wellknown fact that the multiplicity of $V$ in the regular representation $k[G]$ is equal to $\operatorname{dim}(V)$.

Proposition 1.9. We assume that $\operatorname{char}(k)$ does not divide $n$. Let $V$ be a rationally valued irreducible $k$-representation of $G$. Then the multiplicity of $V$ in $N_{G, X}$ and in its dual $\check{N}_{G, X}$ is equal to

$$
\frac{1}{2} \sum_{Q \in Y}\left(\operatorname{dim}(V)-\operatorname{dim}\left(V^{G_{\tilde{Q}}}\right)\right) .
$$

Proof. As in the proof of Corollary 1.3 we write $\langle$,$\rangle for the usual character pairing.$ Then we have:

$$
\begin{aligned}
& \left\langle[V],\left[N_{G, X}\right]\right\rangle=\frac{1}{n} \sum_{P \in X} \sum_{d=0}^{e_{P}-1} d\left\langle[V],\left[\operatorname{Ind}_{G_{P}}^{G}\left(\chi_{P}^{d}\right)\right]\right\rangle \\
& =\frac{1}{n} \sum_{P \in X} \sum_{d=0}^{e_{P}-1} d\left\langle\left[\operatorname{Res}_{G_{P}}^{G}(V)\right],\left[\chi_{P}^{d}\right]\right\rangle \quad \text { (by Frobenius reciprocity) } \\
& =\frac{1}{n} \sum_{P \in X} \frac{e_{P}}{2}\left(\operatorname{dim}(V)-\operatorname{dim}\left(V^{G_{P}}\right)\right) \quad \text { (by Lemma } 1.10 \text { below) } \\
& =\frac{1}{2} \sum_{Q \in Y}\left(\operatorname{dim}(V)-\operatorname{dim}\left(V^{G_{\tilde{Q}}}\right)\right)
\end{aligned}
$$

since over any point $Q \in Y$ there are precisely $\frac{n}{e_{\tilde{Q}}}$ points in the fibre $\pi^{-1}(Q)$ and since $G_{\tilde{Q}^{\prime}}$ is conjugate to $G_{\tilde{Q}}$ for any other point $\tilde{Q}^{\prime}$ in $\pi^{-1}(Q)$. This proves the Proposition for $N_{G, X}$. The same argument applies to $\check{N}_{G, X}$.

Lemma 1.10. Let $C$ be a cyclic group of order c coprime to $\operatorname{char}(k)$, let $V$ be a rationally valued $k[C]$-module, and let $\chi: C \rightarrow k^{\times}$be a primitive character of $C$. Then we have:

$$
\sum_{d=0}^{c-1} d\left\langle\left[\chi^{d}\right],[V]\right\rangle=\frac{c}{2}\left(\operatorname{dim}(V)-\operatorname{dim}\left(V^{C}\right)\right) \quad \text { in } \quad \mathbb{Q} .
$$

Proof. It obviously suffices to consider the case $k=\mathbb{C}$. Since $V$ is rationally valued and $C$ is abelian, the class $[V]$ of $V$ in $K_{0}(\mathbb{C}[C])$ belongs to the image of the canonical homomorphism $K_{0}(\mathbb{Q}[C]) \rightarrow K_{0}(\mathbb{C}[C])$, see the Corollary of Proposition 35, $\S 12.2$, on p. 93 in Serre's book [Se2]. By Exercise 13.1 on pp. 104-105 in [Se2], the permutation representations $\operatorname{Ind}_{H}^{C}\left(1_{H}\right), H$ a subgroup of $C$, form a $\mathbb{Z}$-basis of $K_{0}(\mathbb{Q}[G])$. Since both sides of the formula in the Lemma are additive in $V$, it therefore suffices to prove the Lemma in the case $V=\operatorname{Ind}_{H}^{C}\left(1_{H}\right)$ where $H$ is any subgroup of $C$. Let $h$ denote the order of $H$. Then we obviously have:

$$
\left\langle\left[\chi^{d}\right],\left[\operatorname{Ind}_{H}^{C}\left(1_{H}\right)\right]\right\rangle=\left\langle\left[\operatorname{Res}_{H}^{C}\left(\chi^{d}\right)\right],\left[1_{H}\right]\right\rangle= \begin{cases}1, & \text { if } h \mid d \\ 0, & \text { else. }\end{cases}
$$


Thus we obtain:

$$
\begin{aligned}
& \sum_{d=0}^{c-1} d\left\langle\left[\chi^{d}\right],\left[\operatorname{Ind}_{H}^{C}\left(1_{H}\right)\right]\right\rangle \\
& =h+2 h+\ldots+\left(\frac{c}{h}-1\right) h \\
& =h\left(1+2+\ldots+\left(\frac{c}{h}-1\right)\right) \\
& =h \frac{\left(\frac{c}{h}-1\right) \frac{c}{h}}{2}=\frac{c}{2}\left(\frac{c}{h}-1\right) \\
& =\frac{c}{2}\left(\operatorname{dim}\left(\operatorname{Ind}_{H}^{C}\left(1_{H}\right)\right)-\operatorname{dim}\left(\left(\operatorname{Ind}_{H}^{C}\left(1_{H}\right)\right)^{C}\right)\right),
\end{aligned}
$$

as was to be shown.

Similarly to the deduction of Corollary 1.8 from Corollary 1.7 we deduce the following corollary from Corollary 1.4. An alternative approach to the following corollary based on Ksir's paper $[\mathbf{K s}]$ and Borne's thesis $[\mathbf{B o}]$ can be found in the recent preprint $[\mathbf{J K}]$ by Ksir and Joyner.

Corollary 1.11. We assume that $\operatorname{char}(k)$ does not divide n. Let $D=\sum_{P \in X} n_{P}[P]$ be a $G$-equivariant divisor on $X$ and let $V$ be a rationally valued irreducible $k$ representation of $G$. Then the multiplicity of $V$ in the Euler characteristic $\chi\left(G, X, \mathcal{O}_{X}(D)\right)$ is equal to

$$
\begin{aligned}
& \operatorname{dim}(V)\left(1-g_{Y}\right)+ \\
& \sum_{Q \in Y}\left(\operatorname{dim}(V) m_{\tilde{Q}}-\frac{1}{2}\left(\operatorname{dim}(V)-\operatorname{dim}\left(V^{G_{\tilde{Q}}}\right)\right)+\sum_{d=1}^{l_{\tilde{Q}}}\left\langle\left[\chi_{\tilde{Q}}^{-d}\right],\left[\operatorname{Res}_{G_{\tilde{Q}}^{G}}^{G}(V)\right]\right\rangle\right)
\end{aligned}
$$

where $l_{P} \in\left\{0, \ldots, e_{P}-1\right\}$ and $m_{P} \in \mathbb{Z}$ are given by $n_{P}=l_{P}+m_{P} e_{P}$ for any $P \in X$.

Proof. By Corollary (1.4)(b) we have the congruence

$$
\chi\left(G, X, \mathcal{O}_{X}(D)\right) \equiv-\left[N_{G, X}\right]+\sum_{Q \in Y} \sum_{d=1}^{l_{\tilde{Q}}}\left[\operatorname{Ind}_{G_{\tilde{Q}}}^{G}\left(\chi_{P}^{-d}\right)\right]
$$

in $K_{0}(k[G]) \bmod \mathbb{Z}[k[G]]$. By the Riemann-Roch theorem and the Riemann-Hurwitz formula this congruence becomes an equality in $K_{0}(k[G])$ after adding the term

$$
\left(1-g_{Y}+\sum_{Q \in Y} m_{\tilde{Q}}\right)[k[G]]
$$

on the right hand side. Now using the Proposition 1.9 and Frobenius reciprocity we obtain Corollary 2.11. 
Notice that the multiplicity $\left\langle\left[\chi_{\tilde{Q}}^{-d}\right],\left[\operatorname{Res}_{G_{\tilde{Q}}}(V)\right]\right\rangle$ of the character $\chi_{\tilde{Q}}^{-d}$ of the cyclic group $G_{\tilde{Q}}$ in the restricted representation $\operatorname{Res}_{G_{\tilde{Q}}}^{G}(V)$ can easily be computed. For instance, if $e_{\tilde{Q}}$ is a power of a prime, the following lemma can be applied.

Lemma 1.12. Let $C$ be a cyclic group of prime power order $l^{r}$, let $V$ be a rationally valued $k$-representation of $C$, let $\chi: C \rightarrow k^{\times}$be a primitive character and let $d=l^{s} m \in \mathbb{Z}$ with $s \in\{0, \ldots, r-1\}$ and $m \in \mathbb{Z}$ coprime to $l$. Then the multiplicity $\left\langle\left[\chi^{d}\right],[V]\right\rangle$ of $\chi^{d}$ in $V$ is equal to

$$
\frac{\operatorname{dim}\left(V^{H}\right)-\operatorname{dim}\left(V^{H^{\prime}}\right)}{l^{r-s-1}(l-1)}
$$

where $H$ and $H^{\prime}$ are the (unique) subgroups of $C$ of order $l^{s}$ and $l^{s+1}$, respectively.

Proof. Easy.

\section{The equivariant Grothendieck-Ogg-Shafarevich formula}

The goal of this section is to prove an equivariant Grothendieck-Ogg-Shafarevich formula.

Let $X$ be a connected smooth projective curve over an algebraically closed field $k$ and let $G$ be a finite subgroup of $\operatorname{Aut}(X / k)$ of order $n$. We assume in this section that $\operatorname{char}(k)$ does not divide $n$. Let $\pi: X \rightarrow Y, g_{X}, g_{Y}, G_{P}$ and $e_{P}$ be defined as in $\S 1$. Furthermore, we denote the generic point of $X$ by $\eta$.

Let $l \neq \operatorname{char}(k)$ be a prime and let $\mathcal{F}$ be a constructible $\mathbb{F}_{l}$-sheaf on $X_{\text {ét }}$ with $G$-action, i.e., we have isomorphisms $g^{*}(\mathcal{F}) \rightarrow \mathcal{F}, g \in G$, which satisfy the usual composition rules. Then the étale cohomology groups $H_{\text {ét }}^{i}(X, \mathcal{F}), i \geqslant 0$, are $\mathbb{F}_{l^{-}}$ representations of $G$. Let

$$
\chi_{\text {ét }}(G, X, \mathcal{F}):=\left[H_{\text {êt }}^{0}(X, \mathcal{F})\right]-\left[H_{\text {ét }}^{1}(X, \mathcal{F})\right]+\left[H_{\text {èt }}^{2}(X, \mathcal{F})\right] \in K_{0}\left(G, \mathbb{F}_{l}\right)
$$

denote the equivariant Euler characteristic; here, $K_{0}\left(G, \mathbb{F}_{l}\right)$ is the Grothendieck group of $\mathbb{F}_{l}$-representations of $G$ (of finite dimension). Furthermore, let

$$
\alpha(\mathcal{F}):=\sum_{P \in X} \alpha_{P}(\mathcal{F}) \in \mathbb{Z}
$$

denote the sum of the wild conductors of $\mathcal{F}$ (see p. 188 in $[\mathbf{M i}]$ ) and let $I_{P}:=$ $\operatorname{ker}\left(\mathbb{F}_{l}\left[G_{P}\right] \rightarrow \mathbb{F}_{l}\right)$ denote the augmentation representation of $G_{P}$ (for $P \in X$ ).

The following theorem may be viewed as an analogue of Theorem 1.1; it computes the equivariant Euler characteristic $\chi_{\text {ét }}(G, X, \mathcal{F})$. 
Theorem 2.1 (Equivariant Grothendieck-Ogg-Shafarevich formula). We have in $K_{0}\left(G, \mathbb{F}_{l}\right)_{\mathbb{Q}}$ :

$$
\begin{aligned}
& \chi_{\text {ét }}(G, X, \mathcal{F})= \\
& =\left(\left(2-2 g_{Y}\right) \operatorname{dim}_{\mathbb{F}_{l}}\left(\mathcal{F}_{\bar{\eta}}\right)-\frac{1}{n} \alpha(\mathcal{F})+\sum_{P \in X} \frac{e_{P}}{n}\left(\operatorname{dim}_{\mathbb{F}_{l}}\left(\mathcal{F}_{P}\right)-\operatorname{dim}_{\mathbb{F}_{l}}\left(\mathcal{F}_{\bar{\eta}}\right)\right)\right)\left[\mathbb{F}_{l}[G]\right] \\
& \quad-\sum_{P \in X} \frac{e_{P}}{n}\left[\operatorname{Ind}_{G_{P}}^{G}\left(\mathcal{F}_{P} \otimes I_{P}\right)\right] .
\end{aligned}
$$

Remark 2.2. Let $\operatorname{dim}_{\mathbb{F}_{l}}\left(\mathcal{F}_{P}\right)=\operatorname{dim}_{\mathbb{F}_{l}}\left(\mathcal{F}_{\bar{\eta}}\right)$ for all $P \in X$ with $e_{P} \neq 1$. Then formula (2) has the following shape:

$\chi_{\text {ét }}(G, X, \mathcal{F})=\left(\left(2-2 g_{Y}\right) \operatorname{dim}_{\mathbb{F}_{l}}\left(\mathcal{F}_{\bar{\eta}}\right)-\frac{1}{n} c(\mathcal{F})\right)\left[\mathbb{F}_{l}[G]\right]-\sum_{P \in X} \frac{e_{P}}{n}\left[\operatorname{Ind}_{G_{P}}^{G}\left(\mathcal{F}_{P} \otimes I_{P}\right)\right] ;$

here, $c(\mathcal{F})=\sum_{P \in X} c_{P}(\mathcal{F})$ is the sum of the conductors of $\mathcal{F}$ (see p. 188 in [Mi]). This formula becomes particularly simple in the following two extreme cases.

(a) Let $\pi: X \rightarrow Y$ be étale. Then we have:

$$
\chi_{\text {ét }}(G, X, \mathcal{F})=\left(\left(2-2 g_{Y}\right) \operatorname{dim}_{\mathbb{F}_{l}}\left(\mathcal{F}_{\bar{\eta}}\right)-\frac{1}{n} c(\mathcal{F})\right)\left[\mathbb{F}_{l}[G]\right] .
$$

If $G$ is the trivial group, this is the classical Grothendieck-Ogg-Shafarevich formula (see Theorem 2.12 on p. 190 in [Mi]). In particular, we obtain the following formula for an arbitrary group $G$ :

$$
\chi_{\text {ét }}(G, X, \mathcal{F})=\frac{1}{n} \cdot \chi_{\text {ét }}(X, \mathcal{F}) \cdot\left[\mathbb{F}_{l}[G]\right]
$$

(notice that $n\left(2-2 g_{Y}\right)=\left(2-2 g_{X}\right)$ by the Hurwitz formula). This formula remains valid, if we drop the assumption that $\operatorname{char}(k)$ does not divide $n$ (see the proof below). In particular we obtain that the (non-equivariant) Euler characteristic $\chi_{\text {ét }}(X, \mathcal{F})$ is divisible by $n$. Finally, the latter formula may be viewed as an analogue of Theorem 2.4 in $[\mathbf{E L}]$.

(b) Let $\mathcal{F}$ be the constant sheaf $\mathbb{F}_{l}$ with trivial $G$-action. Then we obtain the following formula:

$$
\chi_{\text {ét }}(G, X, \mathcal{F})=\left(2-2 g_{Y}\right)\left[\mathbb{F}_{l}[G]\right]-\sum_{P \in X} \frac{e_{P}}{n}\left[\operatorname{Ind}_{G_{P}}^{G}\left(I_{P}\right)\right] .
$$

This is the $\mathbb{F}_{l^{-}}$-version of the formula in Remark 2.9 on p. 187 in [Mi]. (Notice that the Artin character is the character of the augmentation representation since $\operatorname{char}(k)$ does not divide $n$.) It can be derived from the $\mathbb{Q}_{l}$-version by applying the decomposition homomorphism as in lines 6 through 9 on p. 191 in [Mi].

Proof. (of Theorem 2.1) As in the proof of Theorem 1.1 we will show that the (Brauer) character values of both sides of formula (2) coincide for all $\sigma \in G$. So let $\sigma \in G$. Then we have: 


$$
\begin{aligned}
& \frac{1}{n} \sum_{P \in X} e_{P} \cdot \operatorname{Trace}\left(\sigma \mid \operatorname{Ind}_{G_{P}}^{G}\left(\mathcal{F}_{P} \otimes I_{P}\right)\right)= \\
& =\frac{1}{n} \sum_{P \in X} \sum_{\tau \in G, \tau^{-1} \sigma \tau \in G_{P}} \operatorname{Trace}\left(\tau^{-1} \sigma \tau \mid \mathcal{F}_{P} \otimes I_{P}\right)
\end{aligned}
$$

(by Lemma (21.28) on p. 509 in [CR2])

$=\frac{1}{n} \sum_{P \in X} \sum_{\tau \in G, \tau(P) \in X^{\sigma}} \operatorname{Trace}\left(\sigma \mid \mathcal{F}_{\tau(P)} \otimes I_{\tau(P)}\right)$

$=\sum_{P \in X^{\sigma}} \operatorname{Trace}\left(\sigma \mid \mathcal{F}_{P} \otimes I_{P}\right)$

$=\sum_{P \in X^{\sigma}}\left(\operatorname{Trace}\left(\sigma \mid \mathcal{F}_{P} \otimes \mathbb{F}_{l}\left[G_{P}\right]\right)-\operatorname{Trace}\left(\sigma \mid \mathcal{F}_{P}\right)\right)$

$=\sum_{P \in X^{\sigma}}\left(\operatorname{dim}_{\mathbb{F}_{l}}\left(\mathcal{F}_{P}\right) \cdot \operatorname{Trace}\left(\sigma \mid \mathbb{F}_{l}\left[G_{P}\right]\right)-\operatorname{Trace}\left(\sigma \mid \mathcal{F}_{P}\right)\right) \quad$ (by Frobenius reciprocity)

$= \begin{cases}-\sum_{P \in X^{\sigma}} \operatorname{Trace}\left(\sigma \mid \mathcal{F}_{P}\right) & \text { for } \sigma \neq \mathrm{id} \\ \sum_{P \in X} \operatorname{dim}_{\mathbb{F}_{l}}\left(\mathcal{F}_{P}\right) \cdot\left(e_{P}-1\right) & \text { for } \sigma=\mathrm{id} .\end{cases}$

Hence, for $\sigma \neq \mathrm{id}$, the character value of the right hand side of formula (2) at the place $\sigma$ equals $\sum_{P \in X^{\sigma}} \operatorname{Trace}\left(\sigma \mid \mathcal{F}_{P}\right)$. By the Lefschetz fixed point formula (see Theorem 2 in $[\mathbf{D o 2}]$ or $[\mathbf{V e}]$; here, we use that $\operatorname{char}(k)$ does not divide $n$ ), this equals the character value of the left hand side.

For $\sigma=\mathrm{id}$, the character value of the right hand side at the place $\sigma$ is

$$
\begin{aligned}
& n\left(2-2 g_{Y}\right) \operatorname{dim}_{\mathbb{F}_{l}}\left(\mathcal{F}_{\bar{\eta}}\right)-\alpha(\mathcal{F})+\sum_{P \in X} \operatorname{dim}_{\mathbb{F}_{l}}\left(\mathcal{F}_{P}\right)-\sum_{P \in X} e_{P} \cdot \operatorname{dim}_{\mathbb{F}_{l}}\left(\mathcal{F}_{\bar{\eta}}\right)= \\
& =\left(2-2 g_{X}\right) \operatorname{dim}_{\mathbb{F}_{l}}\left(\mathcal{F}_{\bar{\eta}}\right)-\left(\alpha(\mathcal{F})+\sum_{P \in X}\left(\operatorname{dim}_{\mathbb{F}_{l}}\left(\mathcal{F}_{\bar{\eta}}\right)-\operatorname{dim}_{\mathbb{F}_{l}}\left(\mathcal{F}_{P}\right)\right)\right)
\end{aligned}
$$

by the Hurwitz formula. By the classical Grothendieck-Ogg-Shafarevich formula (see Theorem 2.12 on p. 190 in [Mi]), this equals the character value of the left hand side at the place $\sigma=\mathrm{id}$.

Thus, the proof of Theorem 2.1 is complete.

The following corollary may be viewed as the analogue of Corollary 1.3; it computes the multiplicity of any irreducible $\mathbb{F}_{l}$-representation $V$ of $G$ in the equivariant Euler characteristic $\chi_{\text {ét }}(G, X, \mathcal{F})$. We write $\operatorname{Irr}\left(G, \mathbb{F}_{l}\right)$ for the set of isomorphism classes of irreducible $\mathbb{F}_{l}$-representations and set $s_{V}:=\operatorname{dim}_{\mathbb{F}_{l}}\left(\operatorname{End}_{\mathbb{F}_{l}[G]}(V)\right)$ for $V \in \operatorname{Irr}\left(G, \mathbb{F}_{l}\right)$. For $Q \in Y$ and $V \in \operatorname{Irr}\left(G, \mathbb{F}_{l}\right)$, let $m_{Q, \mathcal{F}, V}$ denote the multiplicity of the trivial representation $\mathbb{F}_{l}$ in $\mathcal{F}_{P} \otimes I_{P} \otimes \operatorname{Res}_{G_{P}}^{G}(V)$ where $P \in \pi^{-1}(Q)$. 
Corollary 2.3. We assume that $(l, \operatorname{ord}(G))=1$. Then we have in $K_{0}\left(G, \mathbb{F}_{l}\right)_{\mathbb{Q}}$ :

$$
\begin{aligned}
& \chi_{\text {ét }}(G, X, \mathcal{F})= \\
& =\left(\left(2-2 g_{Y}\right) \operatorname{dim}_{\mathbb{F}_{l}}\left(\mathcal{F}_{\bar{\eta}}\right)-\frac{1}{n} \alpha(\mathcal{F})+\sum_{P \in X} \frac{e_{P}}{n}\left(\operatorname{dim}_{\mathbb{F}_{l}}\left(\mathcal{F}_{P}\right)-\operatorname{dim}_{\mathbb{F}_{l}}\left(\mathcal{F}_{\bar{\eta}}\right)\right)\right)\left[\mathbb{F}_{l}[G]\right] \\
& \quad-\sum_{V \in \operatorname{Irr}\left(G, \mathbb{F}_{l}\right)} \frac{1}{s_{V}} \sum_{Q \in Y} m_{Q, \mathcal{F}, \check{V}}[V] .
\end{aligned}
$$

Proof. Let $\langle,\rangle:, K_{0}\left(G, \mathbb{F}_{l}\right) \times K_{0}\left(G, \mathbb{F}_{l}\right) \rightarrow \mathbb{Z}$ denote the symmetric bilinear form given by $\langle[V],[W]\rangle:=\operatorname{dim}_{\mathbb{F}_{l}}\left(\operatorname{Hom}_{\mathbb{F}_{l}[G]}(V, W)\right)$ for any $\mathbb{F}_{l}$-representations $V, W$ of $G$. Then we obviously have:

(a) $\langle[V],[\breve{W} \otimes X]\rangle=\langle[V \otimes W],[X]\rangle$ for any $\mathbb{F}_{l}$-representations $V, W, X$ of $G$.

(b) $\sum_{V \in \operatorname{Irr}\left(G, \mathbb{F}_{l}\right)} \frac{1}{\langle[V],[V]\rangle}\langle[V], x\rangle[V]=x$ for all $x \in K_{0}\left(G, \mathbb{F}_{l}\right)$.

(c) $\left\langle x, \operatorname{Res}_{H}^{G}(y)\right\rangle=\left\langle\operatorname{Ind}_{H}^{G}(x), y\right\rangle$ for any subgroup $H$ of $G, x \in K_{0}\left(H, \mathbb{F}_{l}\right)$ and $y \in K_{0}\left(G, \mathbb{F}_{l}\right)$.

Hence we have for all $P \in X$ :

$$
\begin{aligned}
& \sum_{V \in \operatorname{Irr}\left(G, \mathbb{F}_{l}\right)} \frac{1}{\langle[V],[V]\rangle}\left\langle\left[\mathbb{F}_{l}\right],\left[\mathcal{F}_{P} \otimes I_{P} \otimes \operatorname{Res}_{G_{P}}^{G}(\check{V})\right]\right\rangle[V]= \\
= & \sum_{V \in \operatorname{Irr}\left(G, \mathbb{F}_{l}\right)} \frac{1}{\langle[V],[V]\rangle}\left\langle[V],\left[\operatorname{Ind}_{G_{P}}^{G}\left(\mathcal{F}_{P} \otimes I_{P}\right)\right]\right\rangle[V] \\
= & {\left[\operatorname{Ind}_{G_{P}}^{G}\left(\mathcal{F}_{P} \otimes I_{P}\right)\right] \text { in } K_{0}\left(G, \mathbb{F}_{l}\right) . }
\end{aligned}
$$

Hence we have:

$$
\begin{aligned}
& \sum_{V \in \operatorname{Irr}\left(G, \mathbb{F}_{l}\right)} \frac{1}{s_{V}} \sum_{Q \in Y} m_{Q, \mathcal{F}, \check{V}}[V]= \\
= & \sum_{P \in X} \frac{e_{P}}{n} \sum_{V \in \operatorname{Irr}\left(G, \mathbb{F}_{l}\right)} \frac{1}{\langle[V],[V]\rangle}\left\langle\left[\mathbb{F}_{l}\right],\left[\mathcal{F}_{P} \otimes I_{P} \otimes \operatorname{Res}_{G_{P}}^{G}(\check{V})\right]\right\rangle[V] \\
= & \sum_{P \in X} \frac{e_{p}}{n}\left[\operatorname{Ind}_{G_{P}}^{G}\left(\mathcal{F}_{P} \otimes I_{P}\right)\right] \text { in } K_{0}\left(G, \mathbb{F}_{l}\right) .
\end{aligned}
$$

Thus, Corollary 2.3 follows from Theorem 2.1.

The analogue of the element $\left[N_{G, X}\right]$ occurring in Corollary 1.4(a) is

$$
\sum_{P \in X} \frac{e_{P}}{n}\left[\operatorname{Ind}_{G_{P}}^{G}\left(I_{P}\right)\right]
$$

It is obviously an element of $K_{0}\left(G, \mathbb{F}_{l}\right)$. More generally, $\sum_{P \in X} \frac{e_{P}}{n}\left[\operatorname{Ind}_{G_{P}}^{G}\left(\mathcal{F}_{P} \otimes I_{P}\right)\right]$ is an element of $K_{0}\left(G, \mathbb{F}_{l}\right)$, since $\operatorname{Ind}_{G_{P}}^{G}\left(\mathcal{F}_{P} \otimes I_{P}\right)$ is isomorphic to $\operatorname{Ind}_{G_{P^{\prime}}}^{G}\left(\mathcal{F}_{P^{\prime}} \otimes I_{P^{\prime}}\right)$ for any $P, P^{\prime} \in X$ with $\pi(P)=\pi\left(P^{\prime}\right)$. Furthermore, $\sum_{P \in X} \frac{e_{P}}{n}\left(\operatorname{dim}_{\mathbb{F}_{l}}\left(\mathcal{F}_{P}\right)-\operatorname{dim}_{\mathbb{F}_{l}}\left(\mathcal{F}_{\bar{\eta}}\right)\right)$ is an integer. Thus, Theorem 2.1 implies the following corollary. 
Corollary 2.4. The sum $\alpha(\mathcal{F})$ of the wild conductors $\alpha_{P}(\mathcal{F}), P \in X$, is divisible by $n$.

In particular, Theorem 2.1 expresses $\chi_{\text {ét }}(G, X, \mathcal{F})$ as an integral linear combination of $\mathbb{F}_{l}$-representations; thus, the analogue of Corollary 1.4 is already built into Theorem 2.1.

\section{References}

[BFQ] P. Baum, W. Fulton and G. Quart, Lefschetz-Riemann-Roch for singular varieties, Acta Math. 143 (1979), 193-211.

[Bo] N. Borne, Une formule de Riemann-Roch équivariante pour les courbes, Thèse (Université Bordeaux I, 2000).

[CR2] C. W. Curtis and I. Reiner, "Methods of representation theory, with applications to finite groups and orders", Vol. I, Pure and Applied Mathematics, John Wiley \& Sons, New York (1981).

[Do1] P. Donovan, The Lefschetz-Riemann-Roch formula, Bull. Soc. Math. France 97 (1969), 257-273.

[Do2] P. Donovan, The fixed point formula for equivariant étale sheaves, $J$. Algebra 103 (1986), 427-436.

[EL] G. Eldingsrud and K. Lønsted, An equivariant Lefschetz formula for finite reductive groups, Math. Ann. 251 (1980), 253-261.

[Ha] R. Hartshorne, "Algebraic geometry", Graduate Texts in Mathematics 52, Springer-Verlag, New York (1977).

[JK] D. JoYner and A. KsiR, Representations of finite groups on RiemannRoch spaces, II, preprint (2003), 10 pp.

[Ka] E. KANI, The Galois-module structure of the space of holomorphic differentials of a curve, J. Reine Angew. Math. 367 (1986), 187-206.

[Kö1] B. KöcK, The Lefschetz theorem in higher equivariant $K$-theory, Comm. Algebra 19 (1991), 3411-3422.

[Kö2] B. KöcK, Galois structure of Zariski cohomology for weakly ramified covers of curves, Amer. J. Math. 126 (2004), 1085-1107.

[Ks] A. E. KsIR, Dimensions of Prym varieties, Int. J. Math. Sci. 26 (2001), 107-116.

[Mi] J. S. Milne, "Étale Cohomology", Princeton Mathematical Series 33, Princeton University Press, Princeton (1980).

[Na] S. NAKAJIMA, Galois module structure of cohomology groups for tamely ramified coverings of algebraic varieties, J. Number Theory 22 (1986), 115123.

[Se1] J.-P. SERRE, "Corps locaux", Publications de l'Institut de Mathématique de l'Université de Nancago VIII, Hermann, Paris (1962). 
[Se2] J.-P. SERRE, "Linear representations of finite groups", Graduate Texts in Mathematics 42, Springer-Verlag, New York (1977).

[Ve] J. L. Verdier, The Lefschetz fixed point formula in étale cohomology, in "Proceedings of a conference on local fields" (T. A. Springer, Ed.), Springer-Verlag, Berlin (1967), 199-214.

This article is available at http://intlpress.com/HHA/v7/n3/a6/

Bernhard Köck bk@maths.soton.ac.uk

School of Mathematics

University of Southampton

Southampton SO17 1BJ

United Kingdom 